\title{
Grain size, stress and creep in polycrystalline solids
}

\author{
(C) F.R.N. Nabarro
}

Condensed Matter Physics Research Unit, University of the Witwatersrand, Private Bag 3, WITS 2050, Johannesburg

Division of Manufacturing and Materials Technology, CSIR, P.O. Box 395,

Pretoria 0001, South Africa

(Поступила в Редакцию 29 ноября 1999 г.

В окончательной редакции 11 января 2000 г.)

If a stress $\sigma$ is applied to a polycrystal of grain size $L$, the mode of creep deformation depends on the answers to the following questions: (I) does $\sigma$ exceed the Peierls stress $\sigma_{p}$; (II) does $L$ exceed the dislocation spacing in a Taylor lattice stabilized by $\sigma_{p}$; (III) does $L \sigma$ exceed the value required for a Frank-Read or Bardeen-Herring source to operate within the grain; (IV) does $L^{1 / 2} \sigma$ exceed the Hall-Petch value required for slip to propagate across a grain boundary? The $(L, \sigma)$ plane is thus partitioned into regions in which different modes of creep predominate.

\section{Diffusional Creep and Harper-Dorn Creep}

In diffusional creep, transport of matter occurs by the migration of vacancies from grain boundaries roughly normal to a tensile stress to boundaries roughly parallel to this stress. The migration occurs either through the body of the grain [1,2] or along the grain boundaries [3]. In Harper-Dorn creep, vacancies migrate from edge dislocations with their Burgers vectors roughly parallel to the tensile axis to edge dislocations with their Burgers vectors roughly perpendicular to the tensile axis. The spacing $l$ between adjacent dislocations, which are modelled as forming a Taylor lattice, reaches an equilibrium value such that the stress each dislocation exerts on its neighbour is of the order of the Peierls stress $\sigma_{p}[4,5]$. Thus

$$
b \mu / 2 \pi l \approx \sigma_{p}, \quad l \approx b \mu / 2 \pi \sigma_{p}
$$

and Harper-Dorn creep is possible only if $l<L$, i.e.

$$
L>b \mu / 2 \pi \sigma_{p} .
$$

When this condition is satisfied, the diffusion paths for Harper-Dorn creep are shorter than these for diffusional creep, and Harper-Dorn creep will be faster than NabarroHerring creep provided that [6]

$$
L / b>7 \mu / \sigma_{p} .
$$

Different modes of creep will operate when the product $L \sigma$ is or is not large enough for Bardeen-Herring climb sources to operate within or on the surface of the grain. If the line tension of a dislocation is $\Gamma$, sources can operate freely if

$$
b \sigma>4 \Gamma / L .
$$

With $\Gamma \approx b^{2} \mu / 2$, where $\mu$ is the shear modulus, this becomes

$$
L \sigma>2 b \mu .
$$

Authors of Refs. [7,8] interpret a formula of this kind in the following way. As diffusional creep occurs, edge dislocations climb along the grain boundaries. The inequality (5) represents the condition, that if these dislocations are removed, they can be replaced by new dislocations generated by Bardeen-Herring sources in the grain boundaries. This interpretation seems to be incorrect on two grounds. Firstly, a typical large-angle boundary contains edge dislocations all of the same sign separated by distances of order $b / 3$. If these dislocations all climbed out of the boundary and were not replaced, the total deformation would be of order $30 \%$, larger than that normally observed in diffusional creep. In fact the dislocations will not disappear, but will, statistically, continue to climb in adjacent grain boundaries. Secondly, it is not clear why Bardeen-Herring sources should operate preferentially in grain boundaries. When the inequality (5) is satisfied, sources can operate within the grains, and probably more freely than in the grain boundaries.

The inequality (5) should rather be interpreted in the following way. The equilibrium spacing $l$ of eq. (1) is achieved by a balance between the multiplication of dislocations by the operation of Bardeen-Herring sources within the grain and the annihilation of dislocation pairs under their mutual attraction. This process occurs, and Harper-Dorn creep is possible, if the inequality (4) is satisfied. If the inequality is not satisfied, dislocations climb into the grain boundaries and are absorbed, and, after a possible transient, diffusional creep rather than Harper-Dorn creep occurs.

\section{Stresses above the Peierls Stress}

When the applied stress $\sigma$ exceeds the Peierls stress $\sigma_{p}$,

$$
\sigma>\sigma_{p}
$$

dislocations can move freely by glide.

If, in addition, the inequality (5) is satisfied, dislocations will multiply by glide within the cell much more rapidly than they can annihilate by climb. Harper-Dorn creep gives way to power-law creep [9]. If the product $L^{1 / 2} \sigma$ is less than the Hall-Petch stress-intensity factor $k_{\mathrm{HP}}$

$$
L^{1 / 2} \sigma<k_{\mathrm{HP}},
$$

glide cannot percolate from one grain to its neighbour. 
A possible mode of deformation is then that considered by Spingarn and Nix [10] which may be outlined as follows. The reduced stress is large enough to support glide on only one system in each grain. Coherence between the grains is maintained largely by sliding on the grain boundaries. This sliding is impeded by the ledges formed on the boundaries by pile-ups of dislocations. The rate-controlling process is the smoothing of these ledges by the diffusion of vacancies between adjacent ledges. The distance $\lambda$ between adjacent slip planes in a grain is likely to be of the order of the dislocation passing distance, given by

$$
b \sigma=b^{2} \mu / 2 \pi \lambda
$$

or

$$
\lambda=b \mu / 2 \pi \sigma \text {. }
$$

Allowing for the piling-up of dislocations, the work done by the external stress when a vacancy is transferred from the head of a pile-up is easily seen to be of order

$$
W=L b^{2} \sigma^{2} / 2 \mu \text {. }
$$

On average a vacancy travels a distance $\lambda / 4$ to relieve the local strain, and so the thermodynamic driving force on a vacancy is

$$
4 W / \lambda=4 \pi L b \sigma^{3} / \mu^{2} .
$$

If the effective diffusion constant is $D_{e}$, the flux $\phi$ of vacancy is $D_{e} / k T$ times the thermodynamic force, or

$$
\phi=4 \pi L b \sigma^{3} D_{e} / \mu^{2} k T .
$$

At high temperatures, diffusion will occur through the bulk, $D_{e}$ will be the bulk coefficient of diffusion $D$, and the flow of vacancies at each step will occur through an area of order $L \lambda / 2=L b \mu / 4 \pi \sigma$. The volume $V$ of matter transported at each step in unit time is then

$$
V=L b \mu \phi / 4 \pi \sigma=L^{2} b^{2} \sigma^{2} D / \mu k T .
$$

The time $t$ taken to remove a step is

$$
t=b \lambda L / 8 V=\mu^{2} k T / 16 \pi L \sigma^{3} D .
$$

The shear strain is $b / \lambda$, and so the strain rate $\dot{\varepsilon}$ is given by

$$
\dot{\varepsilon}=b / \lambda t=32 \pi^{2} L \sigma^{4} D / \mu^{3} k T .
$$

At low temperatures, $D$ is replaced by the grain-boundary diffusion coefficient $D_{b}$, and the flux of vacancies occurs over an area of order $L b$. The strain rate is then

$$
\dot{\varepsilon}=128 \pi^{3} L \sigma^{5} D_{b} / \mu^{4} k T .
$$

Both processes occur within the normal range of powerlaw creep.

At higher stresses, several glide systems operate in each grain, and dislocation cells are formed having widths $w$ given approximately by

$$
w=10.5 b \mu / \sigma .
$$

Under these conditions, power-law creep with an exponent 4-5 is observed. As the discussion in Ref. [11] shows, simple mechanisms of creep in this structure lead to the "natural" exponent of 3. An exponent of 5 can be obtained by assuming that diffusion occurs along the cores of dislocations which are present with a density proportional to $\sigma^{2}$, but such a process would have an activation energy only about half the observed value, which is close to that for lattice selfdiffusion. Other models of power-law creep [12,13], which take into account the formation of dislocation cells within the grains, involve rather arbitrary assumptions.

\section{Power-law breakdown}

When the inequality (7) is not satisfied, slip in one grain can transfer to a neighbouring grain. While there is still some thermal activation of the slip process, as is shown by the slow decrease of flow stress with increasing temperature, the rate of deformation is no longer controlled by diffusion, but is a rapidly increasing function of stress. This is the domain of power-law breakdown.

\section{Numerical Values}

The quantities entering this analysis are $b$ and $\mu$, which are well determined, $\sigma_{p}$ and $k$. Both theoretical and experimental values of the Peierls stress $\sigma_{p}$ fall into two classes, one class being some hundred times greater than the other. There are reasons to believe that in problems of progressive plastic deformation it is the values of the lower class which are relevant [14], and we use these. There are no satisfactory theoretical estimates of the HallPetch coefficient $k$, and we use values from the review by Hansen [15].

For aluminium, the relevant parameters are $b=2.86$ $\times 10^{-10} \mathrm{~m}, \mu=26 \cdot 10^{9} \mathrm{~Pa}, \sigma_{p}=2.5 \cdot 10^{-5} \mu$, $k_{\mathrm{HP}}=0.53 \cdot 10^{5} \mathrm{Nm}^{-3 / 2}$, and the relevant map is shown in Fig. 1.

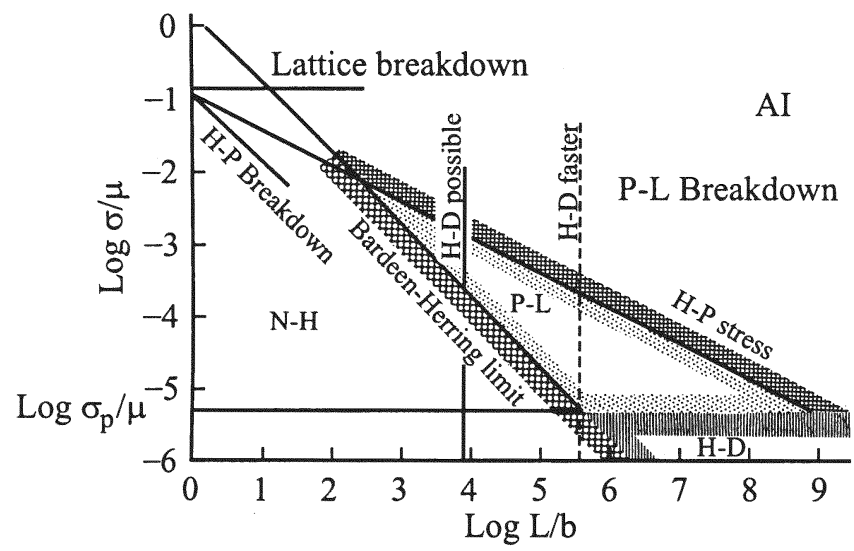

Figure 1. The plane of grain size $L$ and applied stress $\sigma$ is divided into domains of different creep models in aluminium by four boundaries: the Peierls stress $\sigma_{p}$, the grain size at which HarperDorn creep becomes possible (or becomes faster than diffusional creep), the product $L \sigma$ above which Bardeen-Herring (or FrankRead) sources can operate within the grain, and the Hall-Petch product $L^{1 / 2} \sigma$ above which glide can percolate between grains. 


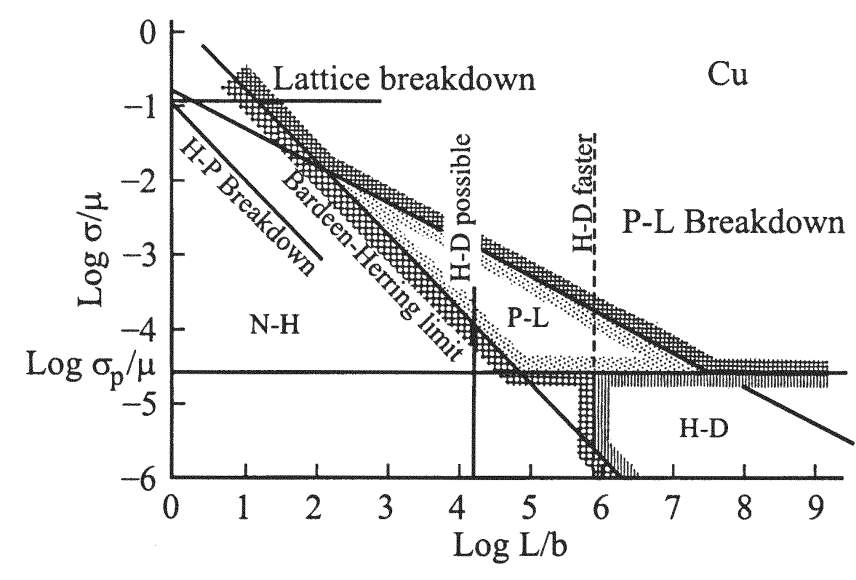

Figure 2. A creep-mode map for copper similar to that of Fig. 1 for aluminium. There is a new domain in which $\sigma<\sigma_{p}$. $L \sigma$ exceeds the Bardeen-Herring limit, but $L^{1 / 2} \sigma$ is below the value at which Harper-Dorn creep is faster than diffusional creep.

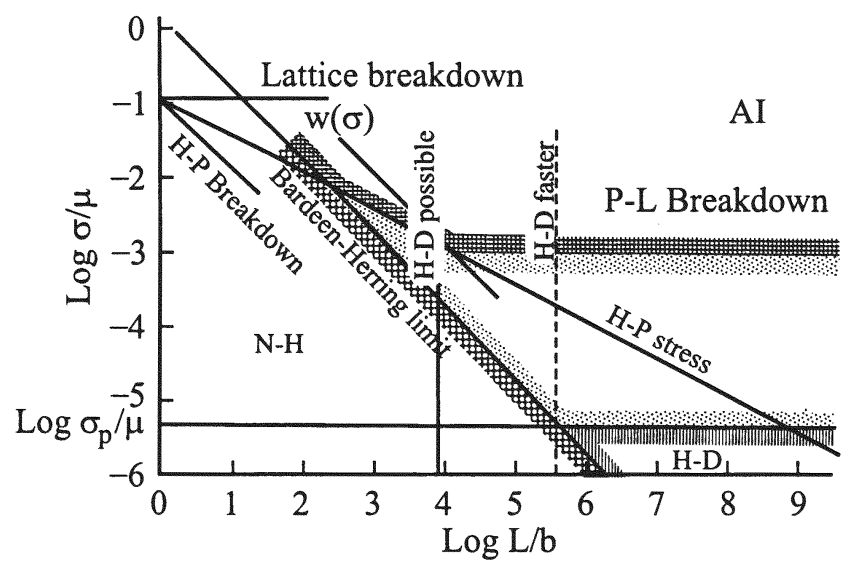

Figure 3. The map for aluminium augmented by the line $w(\sigma)$ determining the dislocation cell size. Where $w<L$, power-law breakdown occurs at a stress independent of grain size.

The present analysis does not apply very high stresses, where the lettice may break down, or at high stresses and very small grain size, where the Hall-Petch criterion may not apply because a pile-up of several dislocations within the cell is not possible. The regions where the analysis does not apply are indicated in the figure.

When $L \sigma$ is below the Bardeen-Herring limit, dislocations cannot multiply within the grain even if $\sigma>\sigma_{p}$. Dislocations are swept into the grain boundaries, and only diffusional creep is possible in the steady state. Above the Bardeen-Herring limit, Harper-Dorn creep occurs when $\sigma<\sigma_{p}$, and the grain size is not too small, even for very large grain sizes where $L^{1 / 2} \sigma$ is large enough to allow dislocations to cross the grain boundary. For $\sigma$ somewhat below $\sigma_{p}$, the Bardeen-Herring limit occurs at about $\log (L / b)=6$, corresponding to $L=290 \mu \mathrm{m}$, in reasonable agreement with the value of $400 \mu \mathrm{m}$ estimated by Mohamed [16] from experimental data. Power-law creep occurs in the region bounded by the Bardeen-Herring limit, the Peierls stress and the Hall-Petch stress line. The region above both the Bardeen-Herring and the Hall-Petch lines is that of power-law breakdown.

For copper, $b=2.56 \cdot 10^{-10} \mathrm{~m}, \mu=48 \cdot 10^{9} \mathrm{~Pa}$, $\sigma_{p}=10^{-5} \mu, k_{\mathrm{HP}}=1.6 \cdot 10^{5} \mathrm{Nm}^{-3 / 2}$, and the resulting map of the $(L, \sigma)$ plane is shown in Fig. 2.

The topology of the map is different from that of Fig. 1. There is a region which lies below the Peierls stress, above the Bardeen-Herring limit, and at grain sizes so small that Harper-Dorn creep is either impossible or slower than diffusional creep. In this new region, diffusional creep will dominate.

\section{Influence of Dislocation Cells}

The discussion so far has assumed that the only obstacles to dislocation motion are the Peierls stress and the grain boundaries. However, dislocations may also assemble into cells of width $w(\sigma)$, where [11]

$$
w(\sigma) \approx 10.5 b \mu / \sigma
$$

and usually do so provided that $w<L$. On the rather drastic assumption that the cell walls are as effective barriers to dislocation motion as are grain boundaries, $L$ must be replaced by $w(\sigma)$ in the preceding discussion. In Fig. 3, the map for aluminium is augmented by the line $w(\sigma)$. At large grain sizes, where the Hall-Petch line lies above the line $w(\sigma)$, the effective grain size is $w(\sigma)$, and powerlaw breakdown occurs at a constant stress given by the intersection of the lines $w(\sigma)$, and $\mathrm{H}-\mathrm{P}$ stress. Then, as is observed, the regimes of Harper-Dorn creep and power-law breakdown are separated by a regime of power-law creep. It appears that this regime covers a factor of several hundreds in stress, in agreement with the observations reported by $\mathrm{Wu}$ and Sherby [17].

\section{References}

[1] F.R.N. Nabarro. Report of a Conference on the Strength of Solids. The Physical Society, London (1948).

[2] C. Herring. J. Appl. Phys. 21, 437 (1950).

[3] R. Coble. J. Appl. Phys. 34, 1679 (1963).

[4] F.R.N. Nabarro. Acta Metall. 37, 2217 (1989).

[5] J.N. Wang. Scripta Metall. et Mater. 29, 1505 (1993).

[6] J.N. Wang. Phil. Mag. A71, 105 (1995).

[7] E. Arzt, M.F. Ashby, R.A. Verrall. Acta Metall. 31, 1977 (1983).

[8] E. Arzt. Acta Mater. 46, 5611 (1998).

[9] J.N. Wang. Scripta Metall. et Mater. 29, 733 (1993).

[10] J.R. Spingarn, W.D. Nix. Acta Metall. 27, 171 (1979).

[11] J.Čadek. Elsevier Materials Science Monographs 48, Amsterdam (1988).

[12] J. Weertman. Trans. ASM 61, 681 (1968).

[13] R.C. Gifkins. J. Austral. Inst. Metals 18, 137 (1973).

[14] F.R.N. Nabarro. Phil. Mag. A75, 703 (1997).

[15] N. Hansen. Metall. Trans. 16A, 2167 (1985).

[16] F.A. Mohamed. Mater. Sci. Eng. 32, 37 (1978).

[17] M.Y. Wu, O.D. Sherby. Acta Metall. 32, 1561 (1984). 